\title{
K3 - A First Look at The New Direct Electron Detection Camera from Gatan Company
}

\author{
Alexander Myasnikov ${ }^{1}$, Shawn Zheng ${ }^{1}$, David Bulkley ${ }^{1}$, Yifan Cheng ${ }^{1,2}$ and David Agard ${ }^{1,2}$ \\ 1. Department of Biochemistry and Biophysics, University of California San Francisco, CA, USA \\ 2. Howard Hughes Medical Institute, University of California San Francisco, CA, USA
}

Much of the success of modern cryo-Electron microscopy is the result of advances in electron detector, specifically the advent of direct electron detectors [1]. Of particular utility has been the Gatan K2 camera that was the first camera to provide single electron detection at practical dose rates, resulting in dramatic improvements in DQE and reduction in noise. The K2 also introduced the concept of superresolution where the electrons can be registered to sub-pixel resolution. Despite the very high internal readout rate of the $\mathrm{K} 2$ camera (400 frames/sec), electron coincidence loss reduces DQE at doses above 3-5 e-/pixel/second, requiring prolonged exposures [2]. The high frame rate does not only increase the DQE, but also allows to correct for beam-induced sample motion by making an alignment of the individual frames before averaging [3], [4].

Here at UCSF Mission Bay we have the opportunity to begin tests on a prototype of the K3, the next generation camera from Gatan. This camera increases the pixel count from 14Mpixels to 24 megapixels $(5,760 \times 4,092)$, resulting in a significantly increased field of view (1.6 X). Moreover it also has a much higher internal readout rate, reducing both coincidence losses and exposure. Together, these promise to substantially improve data collection throughput. The performance and our initial experiences with this new camera will be described during presentation.

\section{References:}

[1] W Kuhlbrandt, Science 343 (2014), p.1443.

[2] X Li et al, Journal of Structural Biology 184 (2013), p. 251.

[3] X Li, Nature Methods 10 (2013), p 584.

[4] SQ Zheng et al, Nature Methods 14 (2017), p 331. 


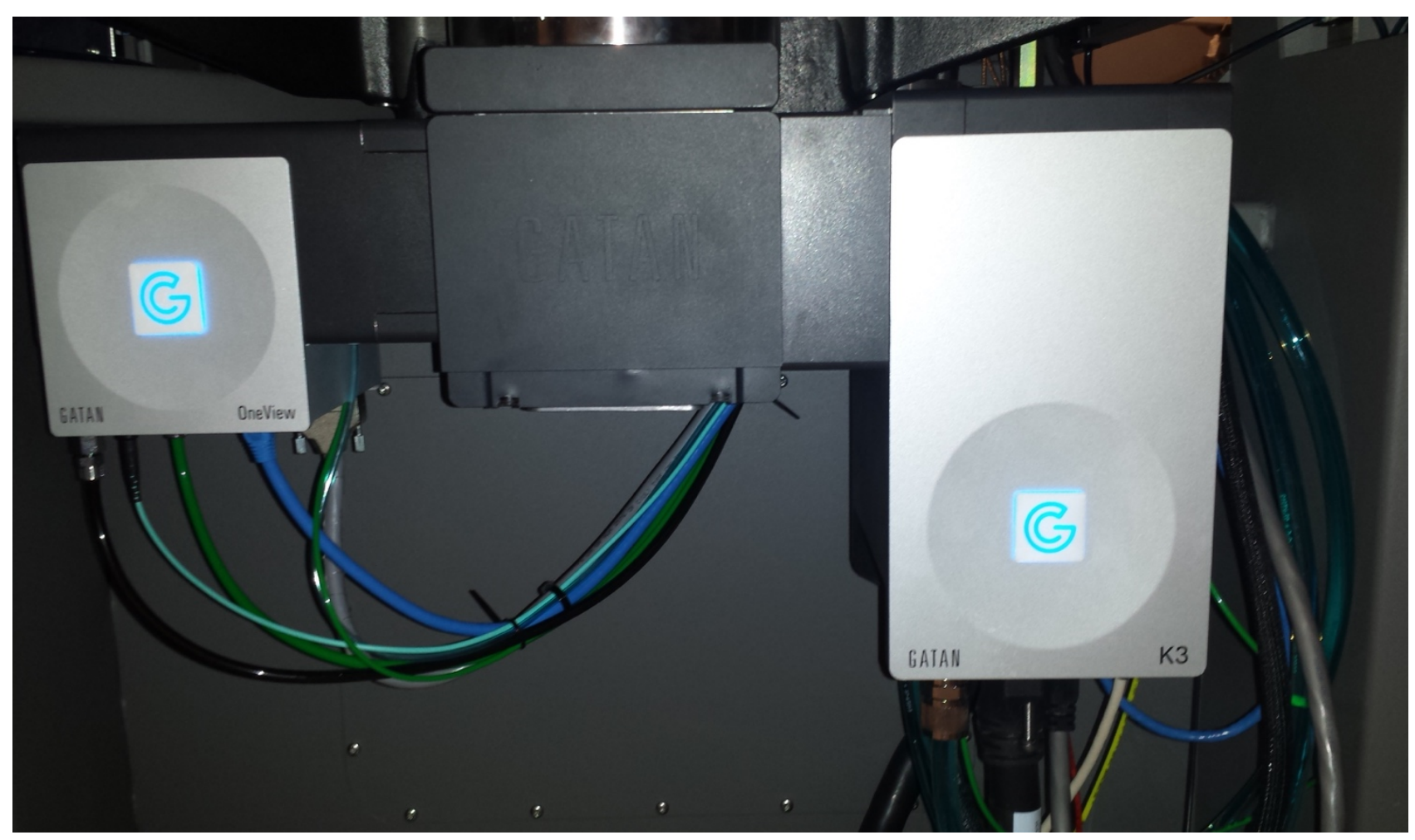

Figure 1. The first Gatan K3 camera installed alongside Gatan OneView Camera 